\title{
Improving the Generation of Decision Objectives
}

\author{
Samuel D. Bond \\ College of Management, Georgia Institute of Technology, Atlanta, Georgia 30308, sam.bond@mgt.gatech.edu \\ Kurt A. Carlson \\ McDonough School of Business, Georgetown University, Washington, DC 20057, kc377@georgetown.edu \\ Ralph L. Keeney \\ Fuqua School of Business, Duke University, Durham, North Carolina 27713, keeneyr@aol.com
}

\begin{abstract}
$\mathrm{R}$ eal decision makers exhibit significant shortcomings in the generation of objectives for decisions that they face. Prior research has illustrated the magnitude of this shortcoming but not its causes. In this paper, we identify two distinct impediments to the generation of decision objectives: not thinking broadly enough about the range of relevant objectives, and not thinking deeply enough to articulate every objective within the range that is considered. To test these explanations and explore ways of stimulating a more comprehensive set of objectives, we present three experiments involving a variety of interventions: the provision of sample objectives, organization of objectives by category, and direct challenges to do better, with or without a warning that important objectives are missing. The use of category names and direct challenges with a warning both led to improvements in the quantity of objectives generated without impacting their quality; other interventions yielded less improvement. We conclude by discussing the relevance of our findings to decision analysis and offering prescriptive implications for the elicitation of decision objectives.
\end{abstract}

Key words: identification of objectives; decision analysis; experimental

History: Received on October 8, 2009. Accepted on January 14, 2010, after 1 revision. Published online in Articles in Advance March 10, 2010.

\section{Introduction}

The only purposeful way that one can influence the future is by making decisions. However, it is not just influence that one desires, but positive influence, and therefore a definition of "positive influence" is required. A broad array of scholars in business, psychology, and behavioral decision research have argued that decisions are best understood as attempts by which people strive to meet their objectives or goals (Drucker 1954, Payne et al. 1988, Austin and Vancouver 1996, Keeney 1992, Higgins 1997, Bettman et al. 1998, Carver and Scheier 1999, Gollwitzer 1999, Morton and Fasolo 2009). In keeping with this tradition, we suggest that for any decision context, the objectives of the decision maker capture the notion of a positive influence on the future.

Objectives represent desires that may be described by a verb and an object (e.g., minimize effort, maximize safety, improve quality). Together, a set of objectives for a decision provides a purpose by specifying what one hopes to achieve. It follows that knowing one's objectives is essential to sound decision making, and this notion is widely accepted by researchers and practitioners of decision science (Raiffa 1968, Smith et al. 1982, Payne et al. 1988, Kirkwood 1997, Leon 1999). Given that objectives provide a foundation for the valuation and comparison of alternatives, the failure to recognize relevant objectives should be viewed alongside oft-cited perceptual and cognitive biases (e.g., Tversky and Kahneman 1981) as a fundamental cause of decision shortcomings.

The preceding logic raises a critical question: When facing a significant decision, do individuals naturally develop a comprehensive set of decision objectives? Based upon an accumulating stream of research, the answer to this question is an emphatic "no." Even for real-world decisions with significant personal consequences, individuals left to their own devices often fail to identify up to half of the objectives that they later acknowledge to be important (Bond et al. 2008). 
The present paper investigates the causes of this failure and also considers potential remedies. Specifically, our purposes are as follows:

1. to explore why decision makers fail to generate many of their relevant objectives, and

2. to develop and test techniques for improving the generation of relevant objectives.

The first purpose requires descriptive research focused on the process of retrieving one's decision objectives, and the second purpose requires prescriptive research focused on helping decision makers do this better. In pursuing the second purpose, we concentrate on techniques that are applicable to any decision and useful with or without the guidance of a professional (e.g., facilitator or consultant).

Our work focuses broadly on the generation of objectives without presuming any knowledge of the decision context. For more specific decision contexts, various prescriptive techniques have been suggested to aid in the generation of objectives. For example, specific preselected alternatives may be used as prompts to generate objectives (Keeney 1992). In other cases, decision makers may be asked to identify their fundamental objectives by reflecting on prespecified attributes (Butler et al. 2006). Many decisions involve the elicitation of objectives from multiple individuals, and in these cases a number of helpful procedures are available, especially when a facilitator is involved (Delbecq et al. 1975, Gregory et al. 1993). Suggestions such as these provide useful supplements to the interventions we explore; however, our contribution is more general and covers a broad array of decision contexts.

The rest of this paper is outlined as follows. Sections 2 and 3 discuss relevant literature on memory and motivation and then build on this discussion to suggest specific reasons that individuals may fail to generate relevant decision objectives. Sections 4 and 5 present empirical tests of these possible reasons and also of potential countermeasures, using decision makers facing decisions of personal consequence. Section 6 summarizes our findings. Section 7 discusses prescriptive implications for those wishing to develop a comprehensive set of objectives for a particular decision context and use this set to enhance the quality of subsequent decisions. Section 8 concludes this paper.

\section{Relevant Research on Memory and Motivation}

A longstanding view among researchers interested in learning and memory is that knowledge representation involves the grouping of similar concepts into categories (Gelman and Markman 1986). A frequently cited demonstration of this principle is the phenomenon of categorical clustering (Bousfield 1953), in which stimuli presented at random tend to be recalled later in terms of categories, even if the categorical relationships are not obvious to an observer (Hudson 1968). This grouping of concepts in memory is generally beneficial, as it facilitates their retrieval and helps individuals bring relevant knowledge to the problem at hand (Medin and Atran 2004). However, cognitive clustering has distinct implications for the elicitation of decision objectives. On one hand, recalling an objective that is highly relevant to an impending decision should facilitate the retrieval of objectives that are conceptually related, many of which will also be relevant. On the other hand, categorical representation is predicated on the existence of conceptual boundaries, the crossing of which requires mental effort. If objectives are stored within memory in a categorical structure-or if they are retrieved in a categorical fashion-then the failure to retrieve certain objectives might stem from a failure to cross category boundaries. As a simple example, many decisions involve subsets of objectives that can be characterized as "short run" or "long run"; e.g., objectives for the construction of a new manufacturing facility may include minimization of construction time, cost, and regulatory hurdles (short run), but also opportunities for later expansion and the ability to adapt to evolving distribution needs (long run). However, diverse research on time-relevant decisions and "temporal construal" indicates that individuals often focus only on either the near future or distant future, to the exclusion of the other (Trope and Liberman 2000). Thus, decision makers tend to identify either shortrun or long-run objectives (but not both) because of an inherent inability to cross the mental boundary that separates these categories of objectives.

Fortunately, the memory literature suggests mechanisms that may enable individuals to cross category boundaries during memory retrieval. First, research based on the categorical clustering phenomenon has 
established that the provision of category names can enhance the recall of items subordinate to those categories (Tulving and Pearlstone 1966). Applied to the task of generating objectives, this finding suggests that giving decision makers a set of specific categories under which to organize their objectives may help them to cross category boundaries and generate a more comprehensive list. Second, the cognitive principle of cue-dependent retrieval states that recall is enhanced by exposure to stimuli that are conceptually related to target items in memory (Tulving 1974). Applied to the generation task, this principle suggests that a more comprehensive set of objectives may be stimulated by presenting decision makers with any material related to those objectives; for example, simply providing a set of "example" objectives may cue the retrieval of other, related objectives (we return to this idea later).

A very different stream of research bearing on the problem at hand deals with motivation and task performance. Many popular theories within this stream link performance outcomes to the motivation of the individual (e.g., Rotter 1966, Bandura 1977, Eccles and Wigfield 2002); details of the these theories vary substantially, but all share the precept that people who believe that they can reach a particular level of performance are more likely to do so. Of particular relevance to present purposes, research suggests that to improve individuals' recall of target items from memory, merely encouraging them to try harder can be an effective motivational technique. In one illustration, Tulving (1966) asked two groups of participants to learn and recall a list of 36 target words. One group simply read the list once and recalled as many words as possible, then repeated this process until the recalled list was complete. A second group also read the list and recalled as many words as possible; however, after becoming "stuck," they were simply asked to recall the list anew; only after becoming stuck three times were they allowed to read the list again. Surprisingly, results indicated that the two groups learned the word list in roughly the same amount of time. Among various reasons for this result, most relevant to our discussion is that many words that seemingly could not be recalled by participants on their first attempt were in fact accessible in memory and retrieved on subsequent attempts.
Applied to the generation of objectives, this discussion suggests that after a decision maker has generated a seemingly "complete" list of objectives, merely asking her to append additional objectives to the list, with the understanding that the current list is incomplete, may encourage the retrieval of a substantial number of previously neglected objectives. Clearly, however, any improvement attained by requesting additional objectives may depend on the specific characteristics of the request. A wide stream within individual and group motivation research suggests that for many different tasks, performance tends to benefit from the provision of highly specific goals that are difficult but attainable (Locke et al. 1981); thus, the most effective requests may be those that ask for a specific and substantial number of additional objectives.

Although the findings discussed thus far are highly relevant to the objective generation process, most of the research focused on a particular paradigm involving the recall of previously unknown lists of items presented to participants by researchers. In contrast, the process of generating one's decision objectives requires the ability of individuals to either recall objectives that already exist in their minds or to construct objectives from more general knowledge. Therefore, we next turn our focus more directly to breakdowns that may occur in the objective generation process.

\section{Why Are Important Objectives Overlooked?}

To address the question, "Why do people fail to generate so many objectives that are in fact important to them?" we used two distinct approaches. The first approach involved a simple survey-based pretest, where participants were confronted with the inadequacy of their generated objectives and asked to introspect on why they had "missed" so many. The second approach utilized insights from this pretest to analyze existing data from Bond et al. (2008). Together, these initial efforts form the foundation for the experiments of $\S \S 4-6$.

\subsection{Pretest: Self-Reported Reasons for Incomplete Objective Lists}

A long-established method for developing hypotheses about cognitive processes underlying a phenomenon 
is to ask individuals directly about their thoughts (Titchener 1912, Lieberman 1979). Therefore, as an initial attempt at identifying causes of objective generation failure, we conducted a pretest using this introspective approach. Participants in the pretest were confronted with their own failure to generate relevant objectives and then asked to provide an explanation. The pretest took the form of a three-page, pen-andpaper survey; participants were students $(N=94)$ at a large southern university who were nominally compensated for their time.

The survey began by providing a decision scenario common to this population: participants were "planning to buy a poster for [their] room or apartment," had visited "an online site that sells thousands of contemporary and classic posters," and needed a means of deciding between the available options. On the first page, participants were asked to "think about and write down all the objectives that matter to you in choosing a poster"; no time limit was imposed. This open-ended method mimics the approach used by Bond et al. (2008), and its validity is supported by various findings reported in that research (e.g., after a five-day delay, participants rated the personal objectives that they had generated using this approach to be more important than either (1) a "baseline" objective generated by others but not themselves or (2) a "bogus" objective that no participants had generated).

After recalling their objectives, participants were presented with a list of 36 different objectives that might reasonably pertain to the decision (e.g., "reflects my sense of taste," "matches room décor," "lifts my mood"). Participants were instructed to identify (with a checkmark) all objectives on the list that were applicable to them personally. On the final page participants were asked to reflect on the discrepancy between the objectives that they had initially listed and those that they had checked: "When people take this survey, they are frequently surprised by how many of their poster objectives they fail to list on the first page. For any of your objectives that you missed on the first page, do you know why this happened?" Six lines were provided for participants to write their responses.

Ten participants did not answer the question, and six stated that their generated list of objectives was complete, leaving 78 usable responses. Through an iterative process, these responses were examined and categorized by the authors, and two distinct "types" of explanation emerged. Approximately 36\% of respondents indicated that their thinking during the generation task was too shallow, that is, they devoted inadequate thought or attention to the decision problem. Specific examples of this type include "lack of thought" and "not thinking it through." Approximately $49 \%$ of respondents indicated that their thinking during the generation task was too narrow, that is, their deliberation was overly focused on a subset of aspects relevant to the decision. Examples included "got too focused and closed my mind around only a few things" and "didn't think about the obvious things." The remaining $15 \%$ of respondents said they could not put objectives into words $(6 \%)$, felt that generation of objectives was unnecessary $(5 \%)$, or did not understand the task $(4 \%)$.

Several insights emerged from this pretest. First, the vast majority of participants (92\%) acknowledged that their generated list of objectives was incomplete. Second, most participants attributed this incompleteness either to lack of thought (i.e., too shallow) or overly focused thought (i.e., too narrow). Finally, only a small minority of participants $(5 \%)$ stated that objectives were unnecessary, even for this relatively simple decision. Given that the studies of $\S \S 4-6$ involve decisions of substantial importance, we are confident that the value of objective generation for those decisions is well understood.

\subsection{Reanalysis: Evidence for Inadequate Breadth and Depth of Thought}

The pretest responses above provide initial evidence that failure in the generation of objectives often results from limitations in either depth or breadth of thinking. To address this issue more systematically, data from Bond et al. (2008) were analyzed as described below. We first present a brief summary of those findings.

Using MBA students, Bond et al. (2008) analyzed three important decision contexts: selection of an MBA program, selection of a weekend executive MBA program, and selection of a summer internship. Figure 1 outlines the general procedure followed (note that the pretest reported earlier and the investigations described in $\S \S 4-6$ employ modified versions of 
Figure 1 Summary of Procedure in Bond et al. (2008)

Step 1: DMs generate as many relevant objectives as they can.

\begin{tabular}{|l|l|}
\hline \multicolumn{2}{c|}{ Objectives list } \\
- & DM Objective A \\
- & DM Objective B \\
- & DM Objective C \\
- & DM Objective D \\
- & $\ldots$ \\
- & $\ldots$ \\
- & $\ldots$ \\
& \\
\hline
\end{tabular}

Step 3: DMs map objectives from Step 1 to the master list. Checked items that map back are selfgenerated objectives; all others are recognized.
Step 2: DMs see the master list and check all objectives that are relevant.

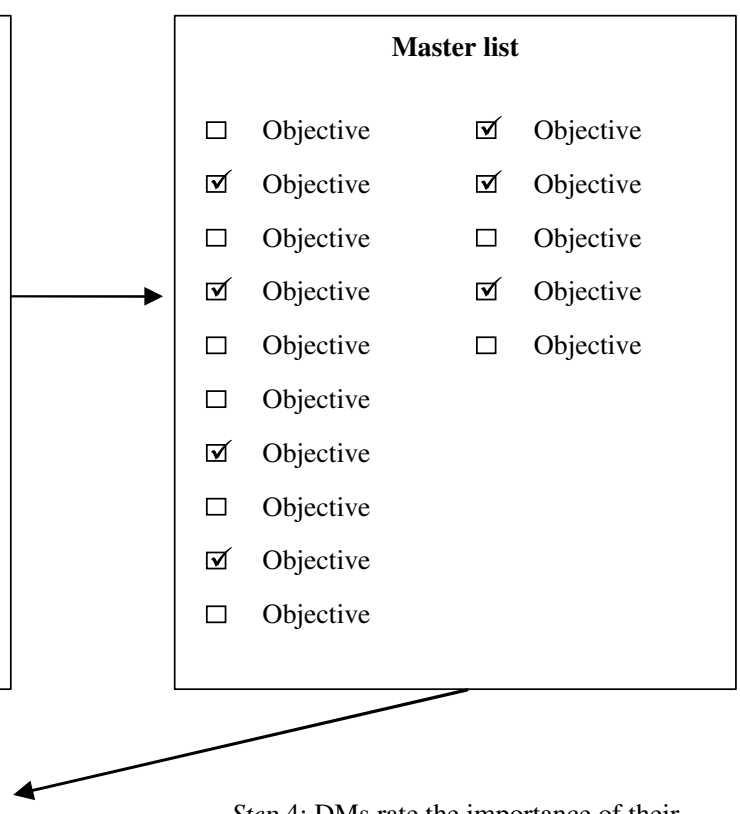

Step 4: DMs rate the importance of their checked objectives.

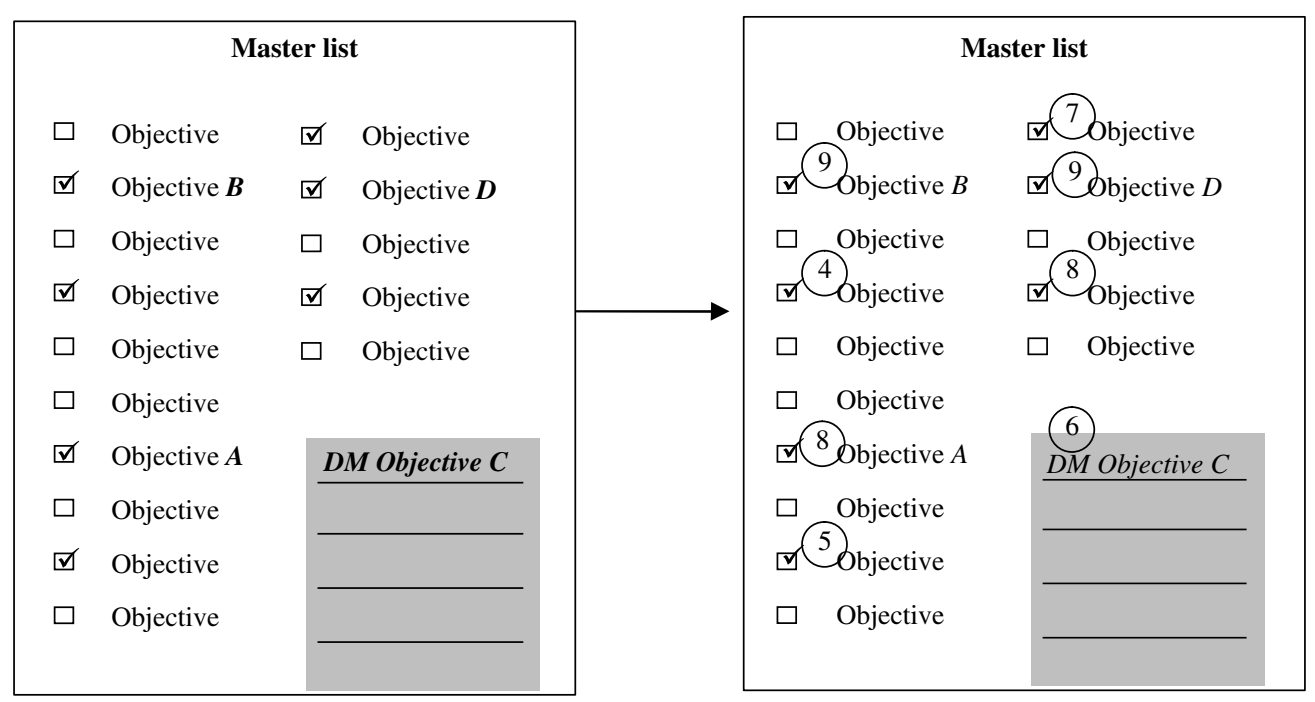

Notes. Studies 1-3 of the current paper expanded on this basic design (see Figure 4). Note that the mapping in Step 3 need not be one to one: multiple items on the self-generated list might map to the same item on the master list, and one item on the self-generated list might map to many on the master list. DM, Decision maker.

this design). Participants first read a short introduction describing the decision context and were then asked to write down all objectives that were personally relevant to this decision (the allotted thinking time varied across studies, from a few minutes to many days). After this generation stage, participants were shown a "master list," designed to contain a mutually exclusive set of virtually all objectives that might reasonably pertain to the decision. Participants were asked to check all the objectives on the 
master list that were personally relevant and to provide importance ratings for each identified objective. Finally, using a participant-guided mapping procedure, the master list of objectives for each participant was partitioned into three subsets: (1) relevant objectives generated without the aid of a master list (we label these self-generated objectives), (2) relevant objectives omitted during the initial generation process but checked on the master list (recognized objectives), and (3) objectives not considered relevant by that individual (i.e., those left unchecked). Note that this procedure obviated the need to interpret the meaning of participants' listed objectives, and it also accounted for the possibility that participants might generate numerous highly similar objectives (these would be mapped into the same objective on the master list and count as only one self-generated objective). By examining the quantity of objectives in the self-generated and recognized subsets, Bond et al. (2008) observed that participants failed to generate a surprising portion of their acknowledged objectives (roughly $50 \%$ across the three studies). Moreover, analyses of importance ratings revealed that self-generated and recognized objectives were strikingly similar in importance, and recognized (missed) objectives were often among the most important.

To determine the extent to which deficiencies in breadth or depth of thinking may have contributed to these findings, we reanalyzed the Bond et al. (2008) data as follows. For each of the three studies, all objectives on the "master list" were subdivided into four distinct categories, such that each category contained objectives that were conceptually similar to one another. For example, in a study involving summer internships, the categories were "tangible benefits during internship," "develop professional skills," "influence job offers after graduation," and "psychological benefits during internship." The categories were mutually exclusive and collectively exhaustive (i.e., each objective appeared in one and only one category). Next, for each participant, the four categories were ordered by the number of self-generated objectives that they contained (i.e., how many objectives the participant generated from that category without the help of the master list).

If objectives are not mentally organized according to categories, then the probability that a checked objective is self-generated should be independent of its conceptual category. Therefore, under this "random generation" model, the proportion of selfgenerated objectives for each ranked category should be the same (specifically, the total number of selfgenerated objectives divided by the total number of objectives checked). Multiplying this proportion by the number of objectives checked in each category, we estimated the number of objectives that would be self-generated under the random model for each individual in each category.

Figure 2 summarizes the results of this analysis. For each of the three studies, the figure presents the following for each ranked category: the total number of objectives checked, the total number of self-generated objectives, and the total number of self-

Figure 2 Breadth and Depth Analysis of the Inadequacy of Generated Objectives

Study $1($ Choice context $=$ Dissertation $)$

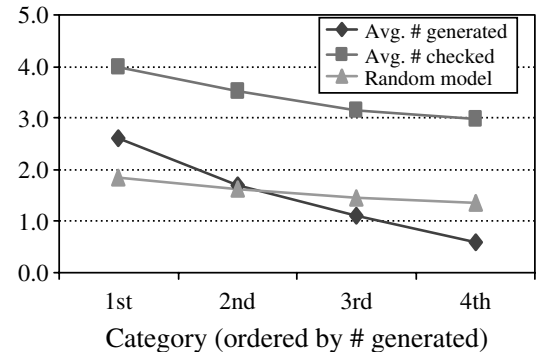

Study 2 (Choice context $=$ MBA program)

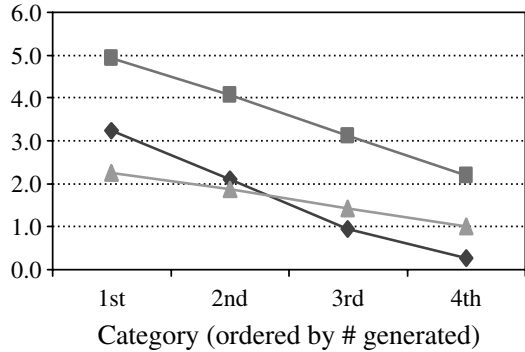

Study 3 (Choice context $=$ Summer internship)

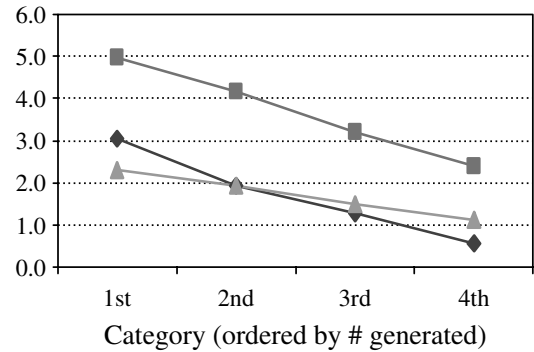

Notes. A reanalysis of data reported in Bond et al (2008) is shown. For each study, the figure shows the average number of self-generated objectives (Avg. \# generated), the average number of recognized objectives (Avg. \# checked), and the number that would be generated according to a "random generation" model. The $x$-axis represents categories of conceptually similar objectives, ranked for each participant according to the number of self-generated objectives. The random model assumes that the proportion (self-generated objectives/total objectives) is the same across all conceptual categories. 
generated objectives predicted by the random generation model. First, we consider the possibility that individuals are thinking too shallowly when generating objectives. If so, the number of self-generated objectives across all conceptual categories (even the highest ranked) should be reliably and substantially lower than the number of checked objectives. Analysis of the data in Figure 2 confirmed this prediction for all three studies.

Second, we consider the possibility that individuals are thinking too narrowly. If so, then certain categories should contain substantially more selfgenerated objectives than expected by the random model, and other categories should contain substantially fewer. Analysis of the data in Figure 2 confirmed this prediction, as higher-ranked (lower-ranked) categories contained significantly more (fewer) selfgenerated objectives than predicted by the random model. For example, in the first study, participants generated an average of 2.60 objectives in their highest-ranked category, considerably more than the number expected if categories did not matter (1.84; $t(61)=9.69, p<0.01)$. However, participants generated an average of only 0.58 objectives in their lowestranked category, considerably fewer than the 1.36 predicted by the random model $(t(61)=-8.56, p<$ $0.01)$. Furthermore, the outperformance in the highestranked category was reliably different from the underperformance in the lowest-ranked category $(t(61)=$ $10.31, p<0.01)$. This pattern replicated across all the studies, strongly indicating that breadth of thinking was insufficient.

Together, the pretest and reanalysis offered tentative evidence that the generation of decision objectives is often hindered by cognitive processing that is insufficient in both depth and breadth. This depthbreadth dichotomy presents a number of interesting parallels to research reviewed in $\$ 2$ on factors influencing recall of lists stored in memory. In particular, many of the techniques shown to aid recall performance can be viewed as tools enabling individuals to think more broadly (e.g., providing categories or examples) or more deeply (e.g., increasing motivation, requesting multiple recall attempts). These insights formed the basis for the experiments below. More generally, our focus on depth and breadth fits within an emerging paradigm that casts cognitive activity as a result of two discrete underlying systems (Stanovich and West 2000, Kahneman 2003, Bond et al. 2009, Milkman et al. 2009): one of these (System 1) is rapid, implicit, emotional, and based on associations, whereas the other (System 2) is slower, conscious, explicit, and analytical. Although System 1 is considered the default in most settings, it is highly susceptible to a range of intuitive biases, such that enhanced System 2 processing often leads to objective improvements in decision quality. To the extent that contemplation of one's objectives involves a substantial amount of reasoned deliberation (alignment of personal values to attributes of the decision, consideration of alternative outcomes, etc.), narrow and shallow thinking may be viewed as deficiencies in System 2 processing, and interventions designed to counter these deficiencies may do so by stimulating the activity of this system.

\section{Interventions to Expand Breadth of Thought}

Under the assumption that inadequate breadth and depth of thinking underlie the failure of decision makers to identify relevant objectives, three empirical studies were conducted to investigate different means of improving the process. The general procedure for all three studies was based on that of Bond et al. (2008), described earlier; Figure 3 provides a schematic overview. Studies 1 and 2 examined the use of a categorical format and the provision of sample objectives as means of encouraging decision makers to think more broadly. Study 3 examined the use of motivational and informational appeals as means of encouraging decision makers to think more deeply. In all three studies, the effectiveness of interventions was measured by their impact on the comprehensiveness of the set of generated objectives.

\subsection{Study 1: Selecting a Dissertation Topic}

Based on the conceptual category analyses of $\S 3$ and the principle that category names enhance recall of items from memory, we surmised that providing decision makers with superordinate objectives categories may stimulate broader thinking and increase comprehensiveness. Therefore, Study 1 tested the effects of a category-based intervention on the objective generation process. As a decision context, the study utilized 
Figure 3 Schematic Overview of Procedures in Each Study

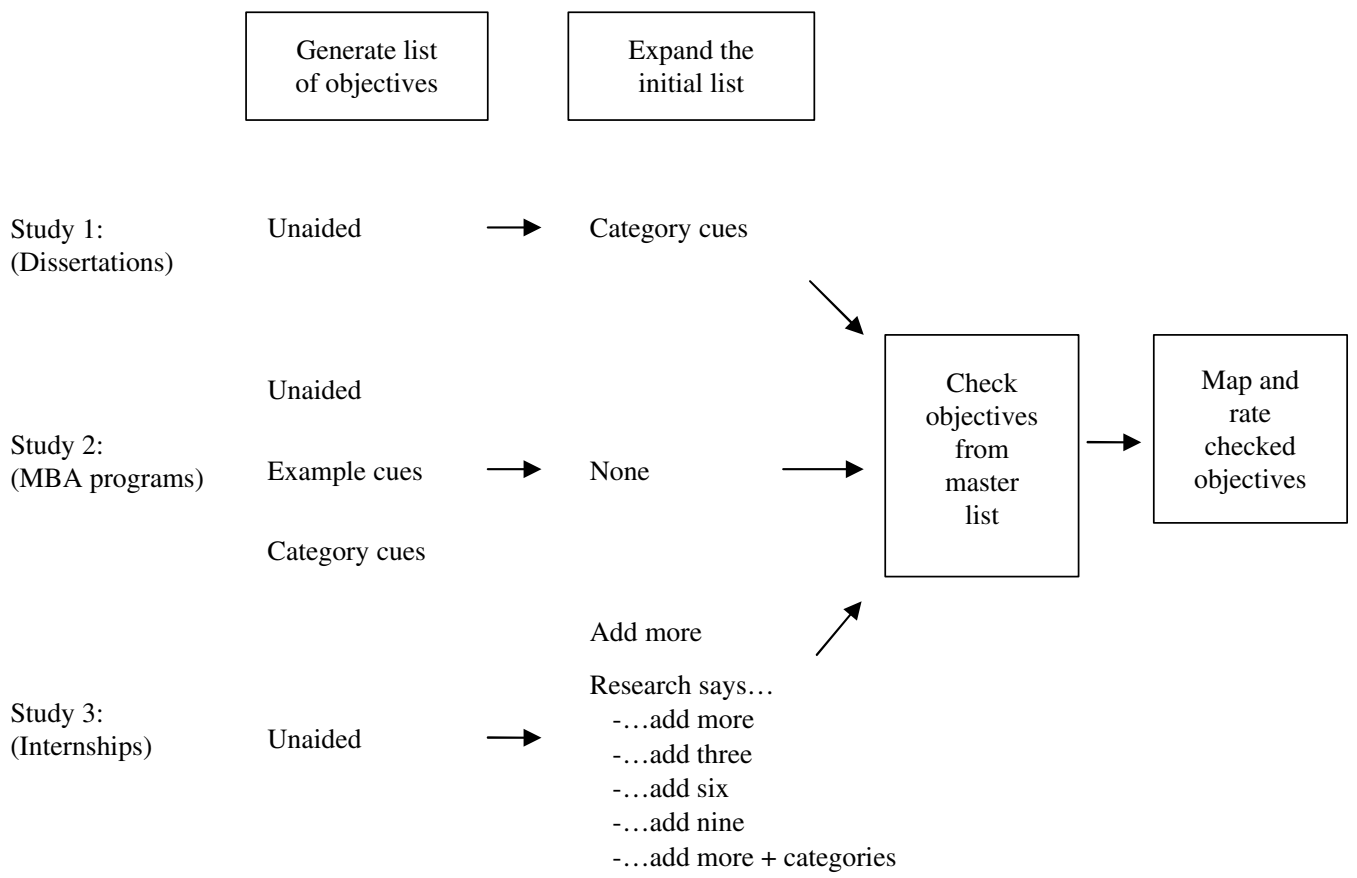

an important, impending decision that is undoubtedly relevant to many of our readers: the choice of a dissertation topic. Because the setup was similar in all three studies, descriptions of Studies 2 and 3 will focus on modifications to the basic design presented here.

4.1.1. Methods. An e-mail recruitment was sent to doctoral students in the department of business administration at a large southeastern university. The recruitment was directed toward students early in the dissertation selection process and highlighted the potential benefits of participation as an aid to this decision. Participants were the 23 students who responded to this request. All participants received the same materials, in the same order (described below). They were compensated for their participation with $\$ 20$ and entry into a drawing for a $\$ 100$ gift certificate.

The study was administered via paper and pencil, and the procedure occurred in two stages. At the first stage, students responding to the recruitment e-mail received a packet containing the objectives questionnaire (seven pages total). After a brief introduction, participants were asked to "think about and write down all the objectives that matter to you in selecting a dissertation topic." The page provided 25 blank lines, numbered 1 to 25 , on which participants could list the objectives that mattered to them.

After completing their initial list, participants were provided an opportunity to expand on their objectives list in a format that encouraged broader thinking: the next page was divided into five categories: "academic objectives while a student," "personal objectives while a student," "professional objectives after graduating," "personal objectives after graduating," and "other." Each category was accompanied by blank lines, each designated by a capital letter, and participants were instructed to "consider each category, and list any additional objectives that matter to you." After adding any additional objectives, participants rated the extent to which they believed their total list of objectives was now complete.

The following page presented a comprehensive "master list" of 34 objectives that might be relevant to the dissertation decision (Figure 4). This list was created by the experimenters through brainstorming and discussion with doctoral students not involved in the study. Sample objectives included "leads to one or more publishable papers," "addresses problems that are important," and "helps me develop myself academically." Beside each objective were two empty 
Figure 4 Master List of Dissertation Objectives for Study 1

\begin{tabular}{|l|l|l|}
\hline II & I & I would like to choose a dissertation topic that... \\
\hline & & Is a topic acceptable to faculty for a doctorate \\
\hline & $\begin{array}{l}\text { Is of interest to a faculty member that I want to serve as my } \\
\text { advisor }\end{array}$ \\
\hline & Leads to multiple publishable papers \\
\hline & Is of interest to me/maintains my interest \\
\hline & Can be scoped/is manageable \\
\hline & Uses methods generalizable to other domains \\
\hline & Is interdisciplinary in nature/combines different areas \\
\hline & Utilizes my academic strengths \\
\hline & Does not require lots of data gathering \\
\hline & Does not require lots of data analysis \\
\hline & Provides opportunity to improve my writing skills \\
\hline & $\begin{array}{l}\text { Addresses issue involving collaboration between public and } \\
\text { private sectors }\end{array}$ \\
\hline & Provides opportunity for sufficient quantitative analysis \\
\hline & Allows for personal time during “dissertation years" \\
\hline & Is of interest to the research/academic community \\
\hline & Includes easily publishable sections \\
\hline & Is enjoyable to do \\
\hline & Helps me develop myself academically \\
\hline & Helps me build a coherent future research program \\
\hline & Helps me balance my career and personal life \\
\hline & $\begin{array}{l}\text { Causes me to learn skills that will be applicable to future } \\
\text { research }\end{array}$ \\
\hline & Improves my ability to write research proposals for funding \\
\hline & $\begin{array}{l}\text { Puts me in control of the dissertation process (e.g., content, } \\
\text { timing) }\end{array}$ \\
\hline & Is innovative/pursues a new idea/novel \\
\hline & Is insightful/has results that weren't obvious prior to my work \\
\hline & Is relevant to real-world application/is implementable \\
\hline & Will help people/organizations make better decisions \\
\hline & Addresses problems that are important \\
\hline & Influences the work of others \\
\hline & Stimulates discussion with colleagues \\
\hline & Provides a basis for further research \\
\hline & Patids in an interesting job talk paper \\
\hline & Prepares me as an independent researcher \\
\hline & & \\
\hline &
\end{tabular}

Notes. Participants identified objectives that were personally relevant by placing a checkmark in the corresponding box of column I. For objectives that mapped to a participant's own initial list, the appropriate number/letter was written in the corresponding box of column II.

boxes. Participants were instructed to put a check in the box adjacent to any objective they believed personally relevant to their selection of a dissertation topic.

Next, participants were asked to examine their listed objectives from the first two pages and compare these listed objectives to their checked objectives on the master list. Any time one of the listed objectives matched an objective on the master list, participants were instructed to write its number or letter in the second box of the objective that it matched. Note that this mapping procedure allowed participants to specify the "meaning" of objectives that they generated; i.e., generated objectives were mapped to checked objectives that the participant perceived as substitutes. The procedure also accounted for differences in the specificity of listed objectives and master list objectives; e.g., a participant who listed a very general objective on the first page might map it to multiple objectives on the master list. Upon completion, participants returned the completed packet to the study administrator at their convenience.

At the second stage, approximately one week later, participants were asked to complete a second packet. The first page of the packet was unique to each participant and listed all the objectives that (s)he had identified as relevant at the first stage. Beside each objective was a line on which participants were asked to "rate each objective in terms of its importance to you," on a scale from 1 (not important) to 9 (of the highest importance). Finally, participants were asked to identify their stage in the dissertation process and were given a set of follow-up questions (gender, year in the program, etc.). Responses to the follow-up questions did not correlate with key results and will not be discussed further.

4.1.2. Results. Three individuals failed to complete the second stage of the study, leaving a sample size of 20. Responses to the dissertation progress question indicated that 15 participants had not yet narrowed their focus to a specific topic, and 5 participants had identified a promising topic. Because results for these two groups did not differ (all ps $>0.10$ ), analyses below cover all 20 participants.

As a preliminary measure, we examined the number of objectives listed by participants at each stage. At the first (unaided) opportunity, participants listed an average of 7.25 objectives for their dissertation. At the second (category-aided) opportunity, participants added an average of 4.5 objectives, a number both reliably different from zero $(t(19)=4.75, p<01)$ and quite large relative to the original 7.25. Only four participants failed to generate any additional objectives. Thus, results from the second (aided) opportunity provide tentative evidence that the generation of objectives benefited from the intervention. 
We next examined the ability of participants to comprehensively generate the decision objectives that were relevant to them. Participants checked an average of 21.9 relevant objectives from the master list. Replicating the results of prior research, less than half of these checked objectives were mapped back to the initial, unaided list; the majority were only recognized after exposure to the master list $(M=9.6$ versus $12.3, t(19)=1.49, p<0.16)$. The fact that participants failed to generate a majority of the objectives they later deemed relevant highlights the potential value of interventions designed to aid the process.

The most important question for present purposes is the extent to which participants benefited from the opportunity to list additional objectives at the second (category-aided) stage. Examination of the mapping results clearly confirmed the benefits of the categories aid. On average, 2.9 of the objectives identified as relevant on the master list were mapped back uniquely to objectives listed at the second (aided) opportunity; again, this number was both reliably greater than zero $(t(19)=5.1, p<0.01)$ and substantial in that the set of self-generated objectives was increased by over $30 \%$.

Having established that the category intervention enhanced the comprehensiveness of the generation process, we examined the relative importance of the added objectives. For the 16 participants who generated additional objectives at the intervention stage, the average importance of the added objectives was 7.3 on the 9-point scale. By comparison, the average importance of objectives mapped to these participants' initial lists was nearly identical: $M=7.7$, $t(15)=1.73, p>0.1$. Therefore, the objectives added by participants as a result of the intervention represented personally significant criteria for their dissertation decision.

4.1.3. Discussion. The results of Study 1 present initial evidence that the generation of decision objectives may be enhanced by external intervention. In the context of an ongoing, consequential decision problem, participants benefited from a focused reflection on their objectives, framed in terms of distinct conceptual categories. These benefits manifested themselves in the generation of additional decision objectives that were substantial in both quantity and importance.
Despite the value of the intervention, however, it was by no means fully successful at enabling participants to generate all of their objectives. Furthermore, the positive effects observed in this study may have arisen from multiple sources; in particular, it is unclear how much of the benefit observed was due to the categories themselves, and how much was due instead to the "second chance" that it afforded participants to contemplate their objectives. Therefore, Study 2 investigated the efficacy of interventions designed to enhance breadth of thinking without appending additional steps to the generation task.

\subsection{Study 2: Choosing an MBA Program}

In Study 2, we examined whether the generation of objectives might be improved without providing a second attempt, by merely altering initial assessment instructions to encourage respondents to think more broadly. In addition, the study extended our investigation to a different decision context: the selection of an MBA program.

To directly compare the effects of our intervention techniques, we created three different versions of instructions for the initial generation task. The standard version was essentially an unaided control condition similar to the first phase of Study 1. The categories version asked participants to think of objectives in terms of specific categories (similar to the second phase of Study 1). The third version, examples, was based on the principle that recall is enhanced by the presence of cues that are conceptually related to target items in memory (Tulving 1974): participants given the examples version were provided four sample objectives from the master list. Although this intervention is intuitively appealing, research on the phenomenon of "part-list cueing" reveals that exposure to items from one portion of a list can actually inhibit recall for other portions (e.g., Nickerson 1984). Hence, it is possible that the provision of examples may not help, or may even hinder the retrieval of other, conceptually dissimilar objectives.

4.2.1. Methods. Participants were 194 students taking part in an MBA orientation program at a large southeastern university. The study was administered in a group setting with a packet of unrelated studies, and $\$ 5$ was donated to charity for each participant. 
Each participant was assigned to one of three conditions: standard, examples, or categories.

The study was administered entirely via paper and pencil, and the stimuli and procedure were similar to that of Study 1, with two major modifications. The first modification was the context of the decision problem: participants were asked to envision that they were planning to pursue an MBA but had not yet decided on a school. Thus, although participants were not actively engaged in the focal decision (as in Studies 1 and 3), they had all undertaken this decision in the recent past. A "master list" of objectives relevant to the MBA decision was created by the experimenters through internal discussion and communication with prior MBA students. The master list contained 28 objectives, including "provides personal growth potential," "availability of electives," and "is in a desirable geographic location."

The second, critical modification involved the creation of three different versions of the initial generation task. Across all three versions, participants were asked to list the objectives that they considered "relevant when making this decision." In the standard condition, these instructions were simply accompanied by a series of blank lines on which to write objectives. In the examples condition, participants were given the same instructions but were also told, "To get you started, we have listed a few examples." The first four lines were not blank, but instead presented four objectives from the master list: "school reputation," "core marketing quality," "school ranking," and "faculty reputation." Finally, participants in the categories condition were given the same initial instructions but told, "To make your task easier, we have created a set of categories." The page was divided into five conceptual categories, accompanied by blank lines: "professional benefits," "professional costs," "personal benefits," "personal costs," and "other." After this stage, all conditions proceeded in the same manner as in Study 1.

4.2.2. Results. Thirty-six participants did not complete all stages correctly, leaving a usable sample of 158. Our discussion will focus on differences across conditions in the comprehensiveness of the generation process, as measured by (1) the raw number of objectives listed and (2) the number of objectives on the master list that were recognized versus selfgenerated.

If either of the generation aids were effective, one might expect a greater number of objectives to be listed by participants in the aided conditions. Analyses revealed that the mean number of listed objectives was 9.2 for the standard condition, 12.7 for the categories condition, and 12.2 for the examples condition (including the four provided objectives). However, because participants in the examples condition were provided four upfront objectives, their performance was artificially inflated. Therefore, we adjusted the number of objectives listed in the examples condition as follows. On average, participants in the standard condition mapped back 2.24 of the four example objectives to their original, self-generated list. Assuming that those in the examples condition would have generated the same number, the lists of these participants were effectively overstated by 1.76 objectives $(4.0-2.24)$. Subtracting this number led to an adjusted average of 10.45 listed objectives.

Univariate analysis of variance (ANOVA) on the number of listed objectives showed an overall effect of condition $(F(2,155)=8.15, p<0.01)$. Follow-up comparisons indicated that the number of objectives listed by the categories condition was reliably higher than that of both the standard condition $(F(1,155)=16.00, p<0.01)$ and the examples condition $(F(1,155)=6.87, p<0.01)$; the latter two did not differ significantly $(p>0.10)$.

When faced with the master list, participants checked an average of 18.0 objectives as relevant. Importantly, this value did not differ among the three generation conditions $(F=2.08, p>0.10)$, indicating that our interventions themselves did not affect assessments of which objectives were relevant. Out of these 18 checked objectives, an average of 9.1 were self-generated, whereas an average of 8.9 were only recognized after exposure to the master list (paired $t(1,157)=0.29$, not significant). As in the previous studies, therefore, participants failed to generate nearly half the objectives that they later identified as relevant.

More critical for present purposes, however, is the extent to which this deficiency differed across the three elicitation formats. The mean number of recognized objectives was 7.9 for the standard condition, 10.8 
for the examples condition, and 7.9 for the categories condition. Analysis by univariate ANOVA indicated that the effect of condition was significant $(F(2,155)=$ $5.39, p<0.01)$. Follow-up comparisons revealed that the number of recognized objectives in the examples condition was reliably higher than that of both the standard condition $(F(1,155)=8.26, p<0.01)$ and the categories condition $(F(1,155)=7.57, p<0.01)$; the latter two conditions did not differ $(p>0.9)$.

4.2.3. Discussion. Study 2 explored the possibility that simple upfront assistance might facilitate the generation of objectives. Two different elicitation formats (examples and categories) were considered as possible catalysts to broader thinking. Results for the examples condition were not optimistic: compared to participants receiving no aid, participants given sample objectives did not list more objectives (after adjusting for the examples provided), and they also checked more "missed" objectives from the master list. In keeping with the principle of part-list cuing, these results suggest that although provision of sample objectives may serve a practical purpose (e.g., by conveying the type of response desired), it may also hinder generation by anchoring respondents on the specific examples provided. Meanwhile, results for the categories condition were mixed: participants given the categorized version listed more initial objectives than unaided participants, but they identified a similar number of "missed" objectives from the master list. In other words, the provision of categories led individuals to generate more specific (but not more complete) sets of objectives. This suggests that framing an initial elicitation in terms of categories may provide value primarily by enabling individuals to think more concretely about their decision objectives.

The results from the categories condition bear directly on the nature of the improvement observed in the second stage of Study 1. If the improvement in that study was due to the provision of categories (and not the "second chance" given to participants to summon their objectives), we should have observed the same improvement in the categories condition of Study 2. Because the improvement in Study 2 was much less substantial, we tentatively infer that the effectiveness of the Study 1 technique did result in part from the "second chance." Our next study explores this issue directly by comparing various techniques designed to increase thinking depth during the generation process.

\section{Study 3: Interventions to Expand Depth of Thought: Choosing an MBA Internship}

Studies 1 and 2 focused on the issue of breadth and provided some evidence that a category-based intervention can assist the generation of objectives. Even in the most successful conditions, however, many objectives were still not identified. Study 3 expands our exploration of potential generation aids by turning to methods that target depth of thinking. We created a set of interventions based in part on the notion that decision makers who believe it is possible to generate more objectives will do so. Furthermore, based on the principle that motivation is enhanced by ambitious but achievable goals (Locke 1981), we reasoned that it should be possible to further improve the process by giving decision makers specific targets for the number of objectives to generate.

Participants in the prior studies were either in the process of making a choice (Study 1) or had recently done so (Study 2). The focal task in this study required MBA students to list their objectives for selecting an internship, and the study was administered in the midst of the internship season. As such, some participants had already made their decision, whereas others had yet to do so. Although our prior results suggested that the ability to generate comprehensive objectives does not depend on the stage of the decision, this study allowed us to examine that question directly.

\subsection{Methods}

Participants were 295 students from an MBA decisionmaking class at a large eastern university. The study was administered over the Internet as a course assignment and was combined with other, unrelated materials. Stimuli and procedure were similar to Study 1 : participants generated an initial list of decision objectives, were provided a second opportunity to do so, then checked, mapped, and rated relevant objectives from a master list. The master list included 29 items, e.g., "provides opportunities to interact with senior managers," "compensates me well," and "enhances my knowledge in a particular industry." However, the 
design of Study 3 included two important modifications. First, the focal decision was that of choosing an MBA internship, and the sample population consisted of two groups: 154 students who were currently engaged in that decision and 141 students who had recently completed the decision. Presumably, both sets of participants were motivated and capable of contemplating the decision and elaborating on their objectives. The second, critical modification involved the use of six distinct interventions described below.

All participants first generated a list of their decision objectives, following instructions similar to those of Studies 1 and 2. Next, each participant was randomly assigned to one of six conditions, each of which corresponded to a different intervention: simple review, deeper review, add three, add six, add nine, and categories. Participants in the simple review condition were asked, "Please review your list of objectives to see if you have missed any objectives, and add any objectives that are appropriate on the lines below." This intervention provided a benchmark against which the others could be compared.

The other five interventions began as follows:

Research and experience [have] clearly demonstrated two important facts about individuals' ability to list their objectives for important decisions. First, people typically list less than $50 \%$ of their significant objectives on the first attempt. Second the objectives that they do not initially list are just as important to them as those they do list. However, there is good news. Research has also found that with additional thought, most people can generate more objectives, and fortunately more of their most important objectives. Indeed, most people can do so without too much extra effort. Hence, we would like you to think a little more deeply about your objectives for your internship decision, and add... on the lines below.

The content of the last sentence diverged across the five interventions. For participants in the deeper review condition, the passage was completed with "any additional personal objectives you develop on the lines below." For participants in the three target conditions (add three, add six, and add nine), the passage was completed with "at least [three, six, nine] more personal objectives you develop on the lines below." Finally, for participants in the categories condition, the passage was completed with the following: "any additional personal objectives you develop on the lines below.
To help you think more broadly, we have provided you with four categories. We ask that you consider each category and try to identify any objectives within it that were omitted from your original list." (The four categories were "tangible benefits," "intangible benefits," "develop/enhance professional skills," and "enhance attractiveness to potential employers.") Participants were provided with 12 lines on which to write their additional objectives.

\subsection{Results and Discussion}

5.2.1. Predecision vs. Postdecision Participants. A comparison of the average number of objectives initially generated by those who had not yet selected an internship $(n=154)$ to those who had already done so $(n=141)$ revealed no reliable difference $\left(M_{\text {undecided }}=\right.$ $\left.6.55, M_{\text {decided }}=6.22, t(293)=0.64, p=0.52\right)$. Additional analyses revealed no reliable difference in the number of objectives added after participants revisited the generation process $\left(M_{\text {undecided }}=3.16\right.$, $\left.M_{\text {decided }}=3.14, t(293)=0.04, p>0.90\right)$. These data strongly suggest that individuals who had recently made a decision were neither more nor less able to generate objectives than are those still engaged in the decision process. Therefore, remaining analyses were conducted on the full sample.

5.2.2. Effectiveness of Interventions. As before, a preliminary measure of comprehensiveness was the number of objectives listed by participants at each stage. At the first (unaided) opportunity, participants listed an average of 6.39 objectives for the internship decision. The number of objectives generated at this stage did not differ by intervention, as expected given that the interventions had not yet occurred $(F(5,289)=149, p=0.192)$.

For each condition, Table 1 depicts the average number of objectives added, the percentage of participants who added at least one objective, and the number of objectives added by those who added at least one. For the number of added objectives, univariate ANOVA indicated significant differences across the six interventions $(F(5,289)=12.84, p<0.01)$, and follow-up comparisons revealed a number of interesting findings. First, participants who received the baseline simple review intervention added very few objectives $(M=0.55)$ relative to those who received 
Table 1 Summary of Intervention Effectiveness in Study 3

\begin{tabular}{lccc}
\hline & $\begin{array}{c}\text { Number of } \\
\text { objectives } \\
\text { added }\end{array}$ & $\begin{array}{c}\text { Percentage of } \\
\text { participants who } \\
\text { added at least } \\
\text { one objective }\end{array}$ & $\begin{array}{c}\text { Objectives added } \\
\text { by those who } \\
\text { added at least } \\
\text { one objective }\end{array}$ \\
\hline $\begin{array}{l}\text { Intervention } \\
\text { Simple review } \\
\text { ("Review your list... } \\
\text { add any") }\end{array}$ & 0.55 & 20.0 & 2.75 \\
$\begin{array}{c}\text { Deeper review } \\
\text { ("Research demonstrates... } \\
\text { add any") }\end{array}$ & 2.77 & 87.7 & 3.16 \\
$\begin{array}{c}\text { Add three } \\
\text { ("Research demonstrates... } \\
\text { add at least three") }\end{array}$ & 3.08 & 86.5 & 3.56 \\
$\begin{array}{c}\text { Add six } \\
\text { ("Research demonstrates... } \\
\text { add at least six") }\end{array}$ & 3.90 & 94.0 & 4.15 \\
$\begin{array}{c}\text { Add nine } \\
\text { ("Research demonstrates... } \\
\text { add at least nine") }\end{array}$ & 4.87 & 86.7 & \\
$\begin{array}{c}\text { Categories } \\
\text { ("Research demonstrates... } \\
\text { add any"-categories) }\end{array}$ & 3.56 & 81.4 & 4.62 \\
\hline
\end{tabular}

Notes. The number of objectives originally listed did not differ across interventions. The average number originally listed prior to any intervention was 6.39.

the deeper review intervention $(M=2.77, t(103)=5.46$, $p<0.01)$. Recall that the only difference between the simple review and deeper review was that the latter reminded participants that objectives are often overlooked and additional effort is often fruitful; hence, the superior performance of deeper review reveals the motivational impact of this message. Furthermore, although many participants in the simple review condition failed to add any objectives, those who did add at least one objective tended to add a substantial number (see Table 1). Together, these results suggest that decision makers must first be convinced that their initial generation is incomplete and motivated to expend additional effort; however, once these requirements are fulfilled, substantial improvement is possible.

All four of the remaining interventions produced substantially more additional objectives than did the simple review (all $p<0.001$ ). The categories intervention produced 3.56 additional objectives, an amount surpassing the simple review intervention but not reliably greater than any others. It is noteworthy that although the categories intervention basically consisted of the deeper review intervention along with suggested categories, the effectiveness of the categories intervention was not reliably higher than that of the deeper review $(t(106)=1.51, p=0.134)$. Hence, for individuals already motivated to think more deeply, the benefit of categories was minimal.

The three target interventions add three, add six, and add nine yielded additional objectives of 3.08, 3.90, and 4.87, respectively. To analyze the effects of specific targets, the number of objectives added was regressed on the number of additional objectives requested. Results revealed a strong positive relationship $(\beta=0.30, F(1,145)=9.27, p<0.01)$, indicating that increasing the target request was an effective means of increasing the number of objectives added.

5.2.3. The Importance of Added Objectives. On average across all individuals, the importance of objectives added after the interventions did not differ from the importance of objectives initially listed $\left(M_{\text {initial }}=7.28, M_{\text {added }}=7.23, t(465)=0.32, p=0.76\right)$. When interventions were examined individually, this null result was also observed for the five most successful interventions: deeper review, add three, add six, add nine, and categories (all $p>0.15$ ). In the simple review intervention, added objectives were rated more important than those originally generated $\left(M_{\text {initial }}=\right.$ 7.37, $\left.M_{\text {added }}=8.41, t(36)=2.16, p=0.037\right)$; however, only six participants in this condition added any objectives at all. In sum, the data strongly indicate that the interventions did not succeed by stimulating trivial objectives; rather, participants induced to think more deeply generated additional objectives that were substantial in both quantity and quality.

\section{Summary of Findings}

The main findings of our empirical investigations can be summarized as follows:

1. Consistent with prior research, individuals facing personally important decisions failed to bring to mind a substantial number of relevant objectives.

2. Providing decision makers with sample objectives did not improve the generation process. This result parallels findings in the memory literature whereby exposure to certain items in a target set may inhibit recall of other items.

3. Providing decision makers with a categorybased aid at the outset of the generation process was of limited success; however, when categories were presented at a second stage along with a request to supplement the original list, an improvement was 
observed. These findings suggest that an important component of successful interventions is the motivation to add more objectives and/or a second chance to think about objectives.

4. The motivation to add more objectives may arise from various sources, but one is the belief that additional effort will be fruitful. This conclusion is most evident in Study 3: simply asking decision makers to add objectives was virtually ineffective, but informing them that research indicates most individuals can add more objectives resulted in substantial improvements.

5. Challenging individuals with a specific level of expected improvement stimulated generation of additional objectives in a manner consistent with motivation theory. As the magnitude of the target increased, the number of additional objectives generated increased as well.

Taken together, these findings support our contention that a large portion of observed deficiencies in the objective generation process can be attributed to insufficient depth and breadth of thinking. Two less central findings also bear mentioning: (i) a template of approximately 30 possible objectives for a decision, even when viewed after successful interventions, allowed individuals to identify many additional relevant objectives, and (ii) the ability of individuals to generate objectives was equivalent for those who had recently made a decision and those currently engaged in that decision.

\section{Prescriptive Implications: Generating Comprehensive Lists of Objectives}

The theoretical and empirical analyses presented in $\S \S 1-6$ suggest several prescriptive insights. In interpreting the advice below, it is important to recognize that a real-world generation task typically involves creating a written list of objectives based on values, information, and knowledge in the minds of individuals. Generating a good set of objectives should enhance understanding and interest in the decision problem and also increase commitment to act.

As a general prescriptive insight, an individual facing a personal decision-or each individual facing a group decision-should first try independently to generate a list of objectives without outside help. This requires little time and contributes in numerous ways to the generation process. Listing objectives helps people appreciate both the difficulty and importance of the task. Reflecting on their lists, most individuals seem to acknowledge that many relevant objectives are missing; the pretest in $\$ 2$ presented various reasons that individuals may give for this deficiency. Having recognized the problem, various techniques may then be used to stimulate the generation of additional relevant objectives.

\subsection{Elicitation Format, Motivation, and Timing}

The most effective interventions in our studies required participants to revisit the task of generating objectives. This finding accords with a burgeoning stream of research suggesting that multiple requests for information from an individual can lead to better estimates than a single request. In problems involving numeric estimation, Vul and Pashler (2008) found that averaging an individual's initial guess with his or her second guess (collected either immediately afterward or three weeks later) produced estimates that were superior to either guess alone. Similarly, Tanner and Carlson (2009) found that asking individuals to predict their future behavior after first asking for an estimate of their "ideal" behavior yielded estimates that were objectively more accurate.

Based on our experiments, one of the most effective ways of inducing decision makers to generate additional relevant objectives is a direct challenge to do so, quantified in terms of a target number of objectives. An appealing aspect of the "challenge" technique is its portability: unlike techniques involving categories, examples, or templates, the provision of a challenge requires virtually no specific decision knowledge. However, the success of the challenge technique depends on communicating to the decision maker that there is a specific, achievable number of objectives they have overlooked. In our experiments, individuals typically generated $30 \%$ to $50 \%$ of their relevant objectives on their first attempt. Assuming that an individual is unlikely to generate all relevant objectives under even ideal circumstances, a reasonable target for the intervention is to approximately double the number of objectives that were initially generated.

Our studies also demonstrated the potential usefulness of a categorical format for eliciting additional 
objectives. For most significant decisions, it may be useful (and would likely not be harmful) to utilize categories to stimulate added thought, providing that concerned individuals have first independently generated as many objectives as they can. One drawback is that the individuals may be required to create relevant categories independently. However, generic categories exist that may apply to many specific decisions (e.g., short- versus long-term objectives, personal versus professional objectives).

For most individual decisions, there is unlikely to be a "master list" of objectives; if such a template does exist, it should be consulted, but only after the individual has independently deliberated about his or her own objectives (for reasons stated above). Persons interested in building an objectives template might challenge themselves to double the number of objectives on their list. They might also follow Benjamin Franklin's classic advice, appending their list over time as they become aware of new objectives (Franklin 2005). To facilitate the process, individuals may wish to identify two or more initial categories of objectives, and then focus on generating specific objectives within category. Keeney (1992) suggests additional methods to broaden and deepen an individual's thinking about objectives, such as using the pros and cons of specific alternatives to stimulate additional objectives. Individuals might also discuss the decision with friends, colleagues, or others facing the same decision to find out what objectives these others consider important. Finally, if an objectives template does exist in some form (e.g., Consumer Reports or an online comparison tool), the individual may conclude by perusing that template and adding relevant objectives.

\subsection{Recommendations for Groups, Policy Makers, and Analysts}

Group decisions often require that more than one person provide objectives for the decision. As each individual will likely identify some objectives not identified by other members, aggregating all members' lists should both broaden and deepen the list of relevant objectives. Our suggestion is to ask each individual independently to develop a list of objectives for the decision, using the guidance offered for individual decisions above. Only after each individual has considered the decision in detail-and attempted to double the number of objectives on his or her initial listshould group discussion occur; the reason for delaying group discussion is that communication may redirect individuals' from their own ideas to those that they hear expressed, limiting the depth and breadth of their thinking. During group discussion, it is important to ensure that participants believe that the set of group objectives includes each member's objectives and also represents their collective interests. Therefore, it may be useful for individuals to take turns and suggest one objective from their list at a time, so that each individual sees and understands his or her influence on the resulting list. The final set of objectives for the group decision should include the union of lists from each individual, with duplicates omitted and similar objectives combined according to a common terminology. In addition, group discussion is itself likely to produce additional objectives that were not originally listed by any individual; these should be included in the final set. Keeney (1999) provides an in-depth case study illustrating many of these principles in an organizational decision setting.

The preceding advice for groups extends to public agencies with responsibility for a decision affecting one or more stakeholder groups. Specifically, we suggest that each stakeholder be asked to generate objectives for the decision following the guidelines above. Next, the objectives of all stakeholders should be combined by the agency responsible for the decision. After duplicate objectives have been omitted and the combined list has been organized, it should be shared with each stakeholder along with documentation indicating how each stakeholder's objectives were accounted for on the combined list.

Finally, for the facilitator or analyst responsible for developing a set of objectives that is accurate and complete, we suggest that each client be guided through the objective generation process, using the guidelines above that are appropriate to the decision. In these settings, a valuable opportunity occurs after client individuals (or groups) have listed their initial objectives. Through discussion with each party, the analyst is able to "drill down" into the reasoning for each listed objective by asking, "Why is this objective important?" Doing so encourages deeper 
thinking and often uncovers new objectives that are fundamental to the decision. An important caveat is that throughout this guided generation process, the analyst must ensure that all respondents believe their views are being heard and will be represented in the resulting list of objectives. Hence, thorough and open communication is essential both during and after the generation process.

Decision objectives may be used for various purposes that enhance the quality of the decision-making process. These purposes include (but are not limited to) the following: clarifying why one cares about the decision, stimulating the creation of alternatives, incorporating multiple stakeholders' views, describing the consequences of alternatives, helping screen out proposed alternatives that are noncontenders, providing a basis for evaluating alternatives that remain, and communicating the pros and cons of the alternatives to relevant parties. Hence, a comprehensive set of objectives provides a solid basis from which to select objectives that will be helpful for any of these purposes. However, only in rare cases would all of the objectives generated be useful for all of the purposes indicated. Instead, decision makers and/or analysts usually must determine the manner in which different objectives will be used. For example, a comprehensive set of objectives for a specific decision might include 30 distinct objectives. From this set, 19 objectives might be useful in creating alternatives, 12 might be particularly relevant for including various stakeholders' concerns, 8 might be considered to screen out obviously inferior alternatives, and 5 may be used in a formal analysis of subsequent contending alternatives. Recent applications including Mild and Salo (2009) and Brothers et al. (2009) have discussed the process of selecting objectives for a formal analysis.

For decision models that formally evaluate alternatives in terms of an objective function (e.g., a multiattribute utility function), only the set of fundamental objectives should be considered to avoid double counting of certain consequences (Keeney 1992). However, as Phillips (1984) discusses in detail, it may not be necessary or appropriate to include all fundamental objectives in the objective function. Initial appraisal may indicate that some fundamental objectives are relatively unimportant, and the implications of other fundamental objectives may be intuitively clear, so that potential additional insights would not justify the time and effort spent including them explicitly in the model.

\section{Conclusion}

Given that decisions are undertaken for the purpose of satisfying underlying objectives, knowledge of one's objectives is fundamental to the decision process. Although it is intuitively appealing that individuals should be capable of independently enumerating their own decision objectives, the current research adds to a growing body of evidence suggesting this is not the case. Fortunately, the interventions described in this paper provide straightforward and actionable methods to improve the generation process by stimulating broader and deeper elaboration of factors relevant to the decision. Moreover, we believe that these interventions represent only the "tip of the iceberg," and we hope that by applying and extending the principles discussed here, researchers and practitioners will uncover a variety of powerful tools to enhance objective generation and improve the quality of resulting decisions.

\section{Acknowledgments}

The authors thank the following colleagues for valuable feedback on earlier versions of this manuscript: James Bettman, Bob Clemen, Robin Gregory, Jay Russo, Jim Smith, and Bob Winkler.

\section{References}

Austin, J. T., J. B. Vancouver. 1996. Goal constructs in psychology: Structure, process, and content. Psych. Bull. 120(3) 338-375.

Bandura, A. 1977. Self-efficacy-Toward a unifying theory of behavioral change. Psych. Rev. 84(2) 191-215.

Bettman, J. R., M. F. Luce, J. W. Payne. 1998. Constructive consumer choice processes. J. Consumer Res. 25(3) 187-217.

Bond, S. D., J. R. Bettman, M. F. Luce. 2009. Consumer judgment from a dual-systems perspective: Recent evidence and emerging issues. N. K. Malhotra, ed. Review of Marketing Research, Vol. 5. M. E. Sharpe, Armonk, NY, 3-57.

Bond, S. D., K. A. Carlson, R. L. Keeney. 2008. Generating objectives: Can decision makers articulate what they want? Management Sci. 54(1) 56-70.

Bousfield, W. A. 1953. The occurrence of clustering in the recall of randomly arranged associates. J. General Psych. 49 229-240.

Brothers, A. J., S. V. Mattigod, D. M. Strachan, G. H. Beeman, P. K. Kearns, A. Papa, C. Monti. 2009. Resource-limited multiattribute value analysis of alternatives for immobilizing radioactive liquid process waste stored in Saluggia, Italy. Decision Anal. 6(2) 98-114.

Butler, J. C., J. S. Dyer, J. Jia. 2006. Using attributes to predict objectives in preference models. Decision Anal. 3(2) 100-116. 
Carver, C. S., M. F. Scheier. 1999. Themes and issues in the selfregulation of behavior. R. S. Wyer, ed. Advances in Social Cognition, Vol. 12. Erlbaum, Mahwah, NJ, 1-105.

Delbecq, A. L., A. H. Van de Ven, D. H. Gustafson. 1975. Group Techniques for Program Planning: A Guide to Nominal Group and Delphi Processes. Scott Foresman, Glenview, IL.

Drucker, P. F. 1954. The Practice of Management. Harper, New York,

Eccles, J. S., A. Wigfield. 2002. Motivational beliefs, values, and goals. Annual Rev. Psych. 53 109-132.

Franklin, B. 2005. Letter to Joseph Priestley, 1772. W. Isaacson, ed. A Benjamin Franklin Reader. Simon and Schuster, New York, 236-237.

Gelman, S. A., E. M. Markman. 1986. Categories and induction in young-children. Cognition 23(3) 183-209.

Gollwitzer, P. M. 1999. Implementation intentions-Strong effects of simple plans. Amer. Psychologist 54(7) 493-503.

Gregory, R., S. Lichtenstein, P. Slovic. 1993. Valuing environmental resources: A constructive approach. J. Risk Uncertainty 7(2) 177-197.

Higgins, E. T. 1997. Beyond pleasure and pain. Amer. Psychologist 52(12) 1280-1300.

Hudson, R. L. 1968. Category clustering as a function of level of information and number of stimulus presentations. J. Verbal Learn. Verbal Behav. 7(6) 1106-1108.

Kahneman, D. 2003. A perspective on judgment and choice: Mapping bounded rationality. Amer. Psychologist 58(9) 697-720.

Keeney, R. L. 1992. Value-Focused Thinking: A Path to Creative Decisionmaking. Harvard University Press, Cambridge, MA.

Keeney, R. L. 1999. Developing a foundation for strategy at Seagate Software. Interfaces 29(6) 4-15.

Kirkwood, C. W. 1997. Strategic Decision Making. Duxbury Press, Belmont, CA.

Leon, O. G. 1999. Value-focused thinking versus alternative-focused thinking: Effects on generation of objectives. Organ. Behav. Human Decision Processes 80(3) 213-227.

Lieberman, D. A. 1979. Behaviorism and the mind-A (limited) call for a return to introspection. Amer. Psychologist 34(4) 319-333.

Locke, E. A., L. M. Saari, K. N. Shaw, G. P. Latham. 1981. Goal setting and task performance-1969-1980. Psych. Bull. 90(1) 125-152.

Medin, D. L., S. Atran. 2004. The native mind: Biological categorization and reasoning in development and across cultures. Psych. Rev. 111(4) 960-983.

Mild, P., A. Salo. 2009. Combining a multiattribute value function with an optimization model: An application to dynamic resource allocation for infrastructure management. Decision Anal. 6(3) 139-152.
Milkman, K. L., D. Chugh, M. H. Bazerman. 2009. How can decision making be improved? Perspectives Psych. Sci. 4(4) 379-383.

Morton, A., B. Fasolo. 2009. Behavioral decision theory for multicriteria decision analysis: A guided tour. J. Oper. Res. Society 60(2) 268-275.

Nickerson, R. S. 1984. Retrieval inhibition from part-set cueing: A persisting enigma in memory research. Memory Cognition 12(6) 531-552.

Payne, J. W., J. R. Bettman, E. J. Johnson. 1988. Adaptive strategy selection in decision-making. J. Exp. Psych.: Learn., Memory, Cognition 14(3) 534-552.

Phillips, L. D. 1984. A theory of requisite decision models. Acta Psychologica 56(1) 29-48.

Raiffa, H. 1968. Decision Analysis: Introductory Lectures on Choices Under Uncertainty. Addison Wesley, Reading, MA.

Rotter, J. B. 1966. Generalized expectancies for internal versus external control of reinforcement. Psych. Monographs 80(1) 1-28.

Smith, J. F., T. R. Mitchell, L. R. Beach. 1982. A cost-benefit mechanism for selecting problem-solving strategies: Some extensions and empirical tests. Organ. Behav. Human Performance 29(3) 370-396.

Stanovich, K. E., R. F. West. 2000. Individual differences in reasoning: Implications for the rationality debate. Behav. Brain Sci. 23(5) 645-665.

Tanner, R. J., K. A. Carlson. 2009. Unrealistically optimistic consumers: A selective hypothesis testing account for optimism in predictions of future behavior. J. Consumer Res. 35(5) 810-822.

Titchener, E. B. 1912. The schema of introspection. Amer. J. Psych. 23 485-508.

Trope, Y., N. Liberman. 2000. Temporal construal and timedependent changes in preference. J. Personality Soc. Psych. 79(6) 876-889.

Tulving, E. 1966. Subjective organization and effects of repetition in multi-trial free-recall learning. J. Verbal Learn. Verbal Behav. 5(2) 193-197.

Tulving, E. 1974. Cue-dependent forgetting. Amer. Scientist 62(1) 74-82.

Tulving, E., Z. Pearlstone. 1966. Availability versus accessibility of information in memory for words. J. Verbal Learn. Verbal Behav. 5(4) 381-391.

Tversky, A., D. Kahneman. 1981. The framing of decisions and the psychology of choice. Science 211(4481) 453-458.

Van de Ven, A. H., A. L. Delbecq. 1974. The effectiveness of nominal, Delphi, and interacting group decision making processes. Acad. Management J. 17(4) 605-621.

Vul, E., H. Pashler. 2008. Measuring the crowd within: Probabilistic representations within individuals. Psych. Sci. 19(7) 645-647.

\section{Correction}

In this version of the paper, "Improving the Generation of Decision Objectives" by Samuel D. Bond, Kurt A. Carlson, and Ralph L. Keeney, originally published online ahead of print March 10, 2010, the first panel heading of Figure 2 was corrected to read "Study 1 (Choice context = Dissertation)," as shown on page 243. 